\section{Glaciations and dense interstellar clouds}

As a possible cause of an ice age on Earth, I have suggested the passage of the Solar System through a region of compression of interstellar matter (ISM) bordering a spiral arm of the Galaxy $^{1}$. Dennison and Mansfield criticise the model because it leads them to expect to find a dense cloud of ISM still very close to us; no such cloud is seen ${ }^{2}$. Their criticism, however, ignores the structure of the Galaxy that provides the basis for my suggestion, and the justification for reviving the idea of a possible relation between ISM and ice ages.

The compression region is a shock region, that is, a 'traffic jam', in the ISM. According to the model, the most recent glaciation was associated with the Sun having been immersed in a cloud of ISM while it traversed such a region. On my interpretation, the Sun emerged from the region after that. Dennison and Mansfield adopt $\sim 20 \mathrm{~km}$ $\mathrm{s}^{-1}$ for the relative speed of the Sun and cloud, and $\sim 10^{4} \mathrm{yr}$ ago for the time of emergence.

Dennison and Mansfield take no account of the fact that the Sun and cloud were travelling through the region with mean speed $\sim 250 \mathrm{~km} \mathrm{~s}^{-1}$ in their orbital motion round the Galaxy. So the place where the Sun emerged from the cloud is more than 10 times further off than in the reckoning of Dennison and Mansfield.

The material of the cloud concerned did indeed emerge from the compression region about the same time, but there are three essential points to recognise: (1) The region forms an oblique shock and, viewed as such, it produces a discontinuity in direction of flow of the ISM passing through it, but not of the stars. After emerging from the region, the cloud material and the Sun have relative motion very different from what they had in it. The trafficjam is like congestion on an escalatorafter people get off an escalator they disperse with relative speeds comparable to the speed of the escalator, not to the relative speed they had while on the escalator. (2) When the material emerges it is no longer compressed, since by definition the compression region is the region where it is compressed. Compression regions themselves are common places of observation, but how the compressed material evaporates out of them is not understood in detail. (3) There is no reason to suppose that clouds retain their individuality as the ISM evaporates from a compression region.

The answer to Dennison and Mansfield is that the dense cloud they expect is not where they infer, it is not dense, and it is not a cloud.

\section{Matters arising}

Matters Arising is meant as a vehicle for comment and discussion about papers that appear in Nature. The originator of a Matters Arising contribution should initially send his manuscript to the author of the original paper and both parties should, wherever possible, agree on what is to be submitted. Neither contribution nor reply (if one is necessary) should be longer than 300 words and the briefest of replies, to the effect that a point is taken, should be considered.

For reasons I have stated briefly elsewhere $\mathrm{e}^{1,3}$, the end of the most recent glaciation could have been the very end of a period of glacial activity that did not even start until after the Sun had emerged from the cloud, and its luminosity had fallen back to normal after an interval of enhancement. Thus the time, $\sim 10^{4} \mathrm{yr}$, is almost certainly an underestimate. In their closing paragraph Dennison and Mansfield go some way towards admitting this further general consideration and its implications. I should take issue with them over several other particular matters were this necessary. It seems, to me, however, that their objections vanish in the light of the foregoing general considerations, and that their discussion actually helps to show that there need be no embarrassing side effects in the model.

I was aware of the problem of a nearby cloud raised by Hoyle and Lyttleton in a paper ${ }^{4} \mathrm{I}$ quoted in ref. 1, and recalled by Dennison and Mansfield, but ideas on the Galaxy and the kinematics of ISM have changed since 1939.

Astronomy Centre,

Iniversity of Sussex,

Falmer, Sussex BNI 9QH, UK

1 McCrea, W. H., Nature, 255, 607-609 (1975).

2 Dennison, B., and Mansfield, V. N., Nature, 261, $32-34$ (1976).

${ }_{4}^{3}$ McCrea. W. H. Observatory, 95, 239-255 (1975), Soc. 35, 405-415(1939).

Dennison and Mansfield REPLy-On encountering a spiral density wave, interstellar matter (ISM) suffers rapid and substantial changes in its flow field and density. Behind the shock, the gas gradually recovers over some millions of years ${ }^{1}$. The actual shock region is extremely narrow, and thus the transit time of the stars and gas through it is negligible in comparison to a million years, the approximate duration of the last general ice age. Behind the shock, the ISM, or any cloud, could not undergo significant changes in velocity, den- sity, or identity caused by spiral density wave in the $\sim 10^{4} \mathrm{yr}$ since the last glaciation, or even in the $\sim 7 \times 10^{-4} \mathrm{yr}$ since the beginning of the last glaciation. To accomplish the rapid changes now proposed by $\mathrm{McCrea}^{2}$, an unjustified assumption of a second abrupt change in the flow field of the ISM behind the initial shock would have to be made. The compression region fades gradually - it does not end abruptly like people leaving an escalator.

Before entering the compression region, the stars and ISM have a small velocity differential of $\sim 10 \mathrm{~km} \mathrm{~s}^{-1}$, because they follow similar orbits. In entering the compression region, the ISM is severely perturbed, while the stars are largely unaffected. This produces velocity differentials between the stars and gas that are an order of magnitude larger than the $5-25 \mathrm{~km} \mathrm{~s}^{-1}$ that McCrea uses. His low velocity differentials are appropriate for regions outside the compression zone. Since the 'traffic jam' applies to the ISM only, it is in the compression zone that there are large velocity differentials, and because the accretion rate in the model depends linearly on the cloud density and inversely as the cube of the velocity differential, the same fractional increase in solar luminosity would now require the frequent encountering of clouds with embarrassingly high densities, from $\sim 10^{8}$ to $\sim 10^{10}$ hydrogen molecules $\mathrm{cm}^{-3}$.

Even if we accept the unorthodox picture that McCrea now uses, an excessive amount of energy is needed to dissipate these dense clouds on time scales of $\sim 10^{4} \mathrm{yr}$. A typical cloud required by the original model has $\sim$ $10^{6}$ hydrogen molecules $\mathrm{cm}^{-3}$ and a diameter of $\sim 1 \mathrm{pc}$. Such dense clouds cool rapidly and cannot store compressional energy. It is easy to show that over a time of $\sim 10^{4} \mathrm{yr}$ a power of $\sim 5 \times 10^{4} L \odot$ is required to overcome the gravitational binding of the cloud. McCrea's present model would require the dissipation of many such clouds throughout the disk of the Galaxy. This requires that on the average a power $\gtrsim$ total luminosity of the Galaxy must be constantly expended in dissipating dense clouds.

This work was partially supported by the National Astronomy and Ionosphere Center which is operated by Cornell University under contract with the NSF. We thank R. Hohlfeld for discussions.

Center for Radiophysics and

Space Research,

Cornell University

Department of Physics and Astronomy, Colgate University

1 Woodward, P. R., Astrophys. J., 195, 61-73 (1975)

2 McCrea, W. H., Nature, 263, $260(1976)$ 